\title{
Researching and Testing the Damping Characteristic and Effect to the Washer of Resinous Cast
}

\author{
Jun WANG ${ }^{1, a,{ }^{*}}$, Juan WANG ${ }^{1, b}$, Guang ZHAO ${ }^{2, c}$, Jun-Cong SU ${ }^{2, c}$ \\ ${ }^{1}$ Wuhan Second Ship Design and Research Institute, No. 450 Zhongshan Road, Wuchang District, \\ Wuhan City, Hubei Province, China \\ ${ }^{2}$ Dalian University of Technology, No. 2 Linggong Road, Dalian city, Liaoning Province, China \\ awj_ship@hotmail.com, bwj9608@sohu.com, ’sjc604@163.com \\ ${ }^{*}$ Corresponding author
}

Keywords: Resinous Cast, Damping Characteristic, Sustaining Bearing, Damping Effect.

\begin{abstract}
In this paper, the damping characteristic of resinous cast material is analyzed first, then theoretic calculation and a series experiments are designed to find damping effect to the joining configuration. The result is the gist to estimate the damping capacity of the new joining configuration, which is the technical base to research the optimum damping joining configuration design between the shafting and hull.
\end{abstract}

\section{Introduction}

According to the requirement of centering and load bearing, the rigid coupling is often used between the shafting and the mounting base of the hull, and the absorber can not be installed, so it's short of an effective measure in vibration attenuation and vibration isolation. The main purpose of analyzing and testing to the connection of resinous cast gasket between the shafting and the mounting base of the hull is to evaluate the noise reduction performance which the new damping vibration attenuation coupling structure has been used.

The damping vibration attenuation is transforming the vibration energy to the thermal energy and spread them out, so as to achieve purpose of vibration attenuation. The method is to rely on improving mechanical structural damping to reduce or eliminate the noise that produced by mechanical vibration and improve the dynamic stability of machinery. The most important indicator that measures the damping effect of structural damping is the loss factor or the vibration transfer ratio of the system. If only depend on the inherent damping characteristics of structure and energy dissipation which is made in the general method on structure of the node, the effect is not obvious, and the vibration response of the equipment will be the general typical response. But it will be an obvious effect if adopting the method of structural damping vibration attenuation with damping technology to decrease the vibration amplification when resonance of structure of equipment is happened.

First in this paper, analyzing the damping characteristics and service conditions of resinous cast gasket itself, and establishing physical mathematical model under the condition of reasonable assumption to describe the connection between resinous cast gasket and the related factors, optimize parameters and design structure, and define the structure size of resinous cast gasket on different shaft system equipments reasonably. Modeling, analyzing and calculating the transfer characteristic of the radial force of dynamic with finite element method. Research the damping effect of the middle bearing in the shaft with the resinous cast gasket, and compare with the test results, to provide reference for the design that reduce the transmission of noise vibration from shaft equipment to the hull.

\section{Damping Characteristics Analysis of Resinous Cast Gasket}

Using losing factor $\eta$ to represent the size of material damping, defining as the ratio of the energy dissipation and the Maximum elastic potential energy of system during the phase change of per unit radian. It shows that when structure vibrates, the size that vibration energy transform into heat during per unit time. The bigger thenis, the better the damping characteristics is. 
According to the free vibration attenuation, the damp time domain graph of resinous cast material has been figured out. And the natural frequency and damp coefficient graph of specimens has been figured out after the spectral analysis, as shown in Fig.1 Fig.2.

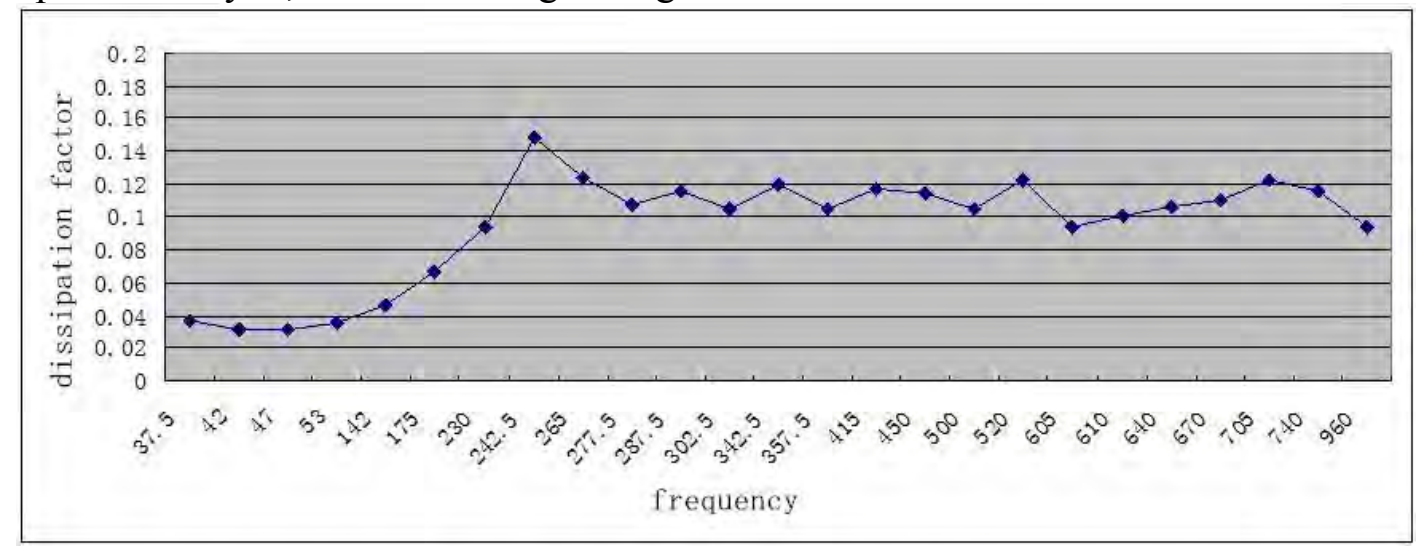

Fig.1 Damp Coefficient Graph of Resinous Cast Material

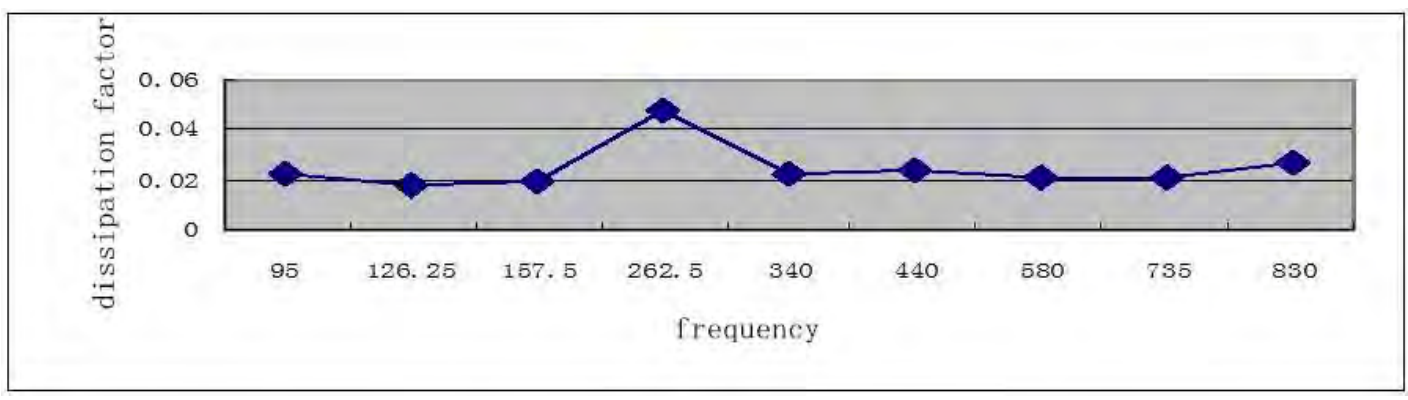

Fig. 2 Damp Coefficient Graph of Steel Material

According to Fig.1 and Fig.2, it is obvious that the damping characteristic of resinous cast gasket is better than steel gasket. According to the Test method of measurement of internal damp coefficient and the attenuation law of material damp coefficient (it is tend to smooth in high frequency), it can be inferred that the damping characteristics of resinous cast gasket is more stable in higher frequency range than $1000 \mathrm{~Hz}$.

\section{Modeling and Analysis of Shafting Steady Bearing on the Ship}

The shafting steady bearing is one of the main bearing components of the ship propulsion shafting. Besides bearing the shafting gravity, it is the main way to transmit the lateral disturbing force when shafting rotates to the hull structure. It is an effective way to reduce the force transition efficiency, which has important significance for damping vibration and noise of the hull. In this work, using finite element analysis method for existing test bench using two different types of steady bearing coupling structure, which uses the connection of steel gasket and resinous cast gasket. Modeling and calculating the transfer characteristics of its dynamic radial force, and the results were preliminary comparative analysis.

\section{Mathematical Analysis Equation}

Damping Equation. For the structural damping system, the equation of motion is:

$$
m \ddot{x}+j h x+k x=F e^{j \omega t}
$$

Or

$$
m \ddot{x}+k(1+j \eta) x=F e^{j \omega t}
$$

In the equation, the $(j h x)$ is damping force, the $k(1+j \eta)$ is complex stiffness, the $h$ is constant, and the $\eta=h / k$ is called loss factor. 
The equivalent viscous damping coefficient of the system is:

$$
C_{e q}=h / \omega=\eta k / \omega=\eta \sqrt{m k}
$$

In the equation, the $\omega$ is natural frequency.

So loss factor of the structural damping system is:

$$
\eta=\frac{c_{e q}}{\sqrt{m k}}=2 \zeta_{e q}
$$

Or

$$
\zeta_{e q}=\frac{1}{2} \eta
$$

In this equation, the $\zeta_{e q}$ is the equivalent viscous damping ratio of the structural damping system, which is the damping ratio of input while the finite element analysis is carrying out on the resinous cast gasket.

Transfer Rate and Isolation Effect of the Exciting Force. The force transfer rate TR is defined as the ratio of the transfer force FT and exciting force F0, that is:

$$
T_{R}=\frac{F_{T}}{F_{0}}
$$

The isolation rate of transfer force $\lambda$ is:

$$
\lambda=\frac{F_{T}^{G}-F_{T}^{H}}{F_{T}^{G}} \times 100 \%
$$

In this equation, the $F_{T}^{G}$ and $F_{T}^{H}$ are the maximum peak transfer force of steel and resinous cast gasket structure.

The isolation efficiency of transfer force $\beta$ is:

$$
\beta=20 \log \frac{F_{T}^{G}}{F_{T}^{H}} \quad(d B)
$$

\section{Inite Element Modeling of Steady Bearing}

When establishing a dynamic model for steady bearing, considering that the shape of the bearing itself is complex and the effect of inertia torque affect to the system, modeling with solid model unit, and simplifying the shape of the bearing slightly, so that the weight that has been simplified is equal to the actual weight, bearing and pedestal are all using solid element SOLID45, as shown in Fig.3.

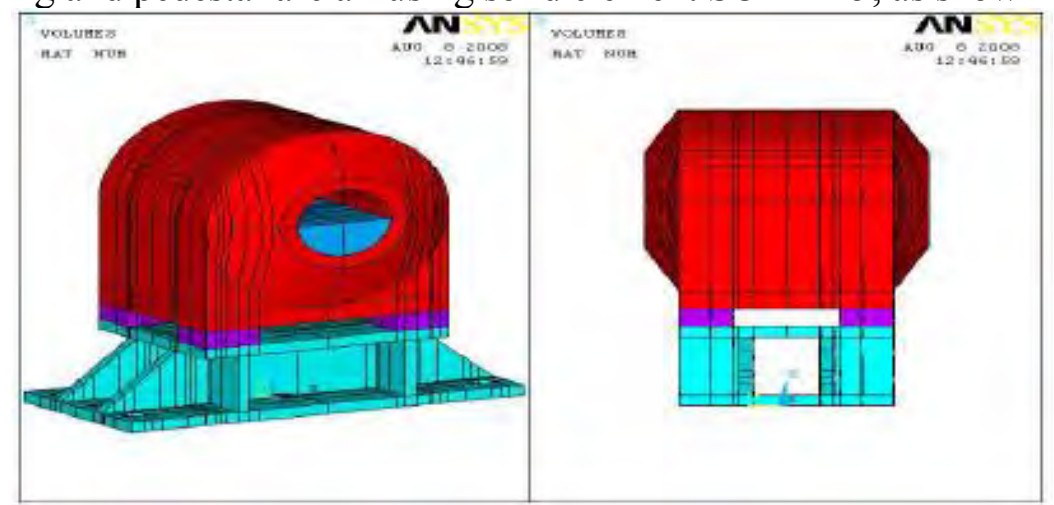

Fig. 3 Entity Model of Sustaining Bearing and Pedestal 
In order to figure out the force transfer rate of the bearing house, it need to apply simple harmonic exciting force within the frequency range of interest to the bearing house, to make the force on the bearing house as close to the actual state. To this end, in this model, establishing a semi-cylindrical, rigid massless false axis which is equal in length with the bearing in the bush, and the lower part close ties to the bearing inner surface. Exciting forces is perpendicular applied to the central axis in the form of a concentrated load, and effect on the bearings through a rigid coupling node between the shaft and the bearing. In order to calculate the reaction force, set fixed bearing on all nodes of the underside of the bearing house. The sum of all these vertical reaction force of the fixed bearings is transfer force of the system.

In this calculation, the bolt, which connects the bearing house and the base, is equivalent to the spring with combin14. Computing model is divided into two kinds: 1) Model 1 - intermediate gasket with steel material; 2) Model 2 - intermediate gasket with resinous cast material.

In these two models, the bearings and the base use the same steel material, which characteristic parameter values as follow:

(1)Steel material:

The elasticity modulus E=210 GPa;The density $\rho=7800 \mathrm{~kg} / \mathrm{m} 3$; The Poisson's ratio $\mu=0.3$.

(2)Resinous cast material:

The elasticity modulus E=5.4 GPa;The density $\rho=1620 \mathrm{~kg} / \mathrm{m} 3$; The Poisson's ratio $\mu=0.337$.

(3)Bolt:

The elastic stiffness $\mathrm{k}=1.413 \mathrm{e}^{9} \mathrm{~N} / \mathrm{m}$; The finite element cell division of the two models is the same, which is divided into 9984 nodes, 9984 units.

\section{Result and Analysis}

In the calculation, a sinusoidal excitation $1 \mathrm{~N}$ is applied along the vertical at the midpoint (here $\mathrm{Y}$ direction) in massless rigid fake axis.

The calculation result of force transfer rate of the two models is as shown in Fig.4. Represent the calculation results of steel and resin gasket.

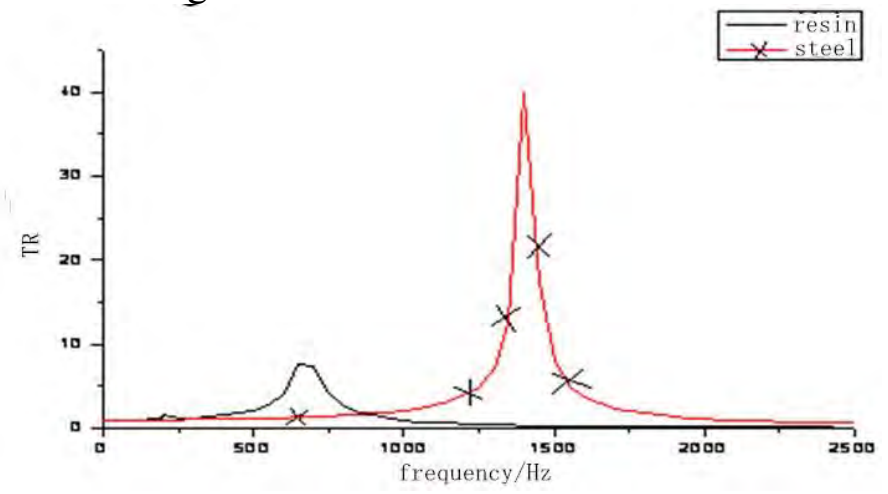

Fig. 4 Contrastive Curve of Force Transmitted Efficien

In order to evaluate the isolation that the excitation force effect the bearings which have been used the resinous cast gasket, we compare the maximum peak transfer force of two structures in the whole frequency band, and get the isolation rate $\lambda$ and isolation efficiency $\beta$ value, as shown in Tab.1.

Tab.1 Damping Efficiency of Transmitted Force

\begin{tabular}{|c|c|c|c|c|}
\hline $\begin{array}{c}\text { Gasket } \\
\text { materials }\end{array}$ & $\begin{array}{c}\text { Peak } \\
\text { frequency(Hz) }\end{array}$ & $\begin{array}{c}\text { Maximum } \\
\operatorname{peak}(\mathrm{N})\end{array}$ & $\begin{array}{c}\text { Isolation } \\
\operatorname{rate}(\lambda, \%)\end{array}$ & $\begin{array}{c}\text { Isolation } \\
\text { efficiency }( \\
\mathrm{dB})\end{array}$
\end{tabular}

As it is shown in fig.4, in a low frequency band of $0 \sim 100 \mathrm{~Hz}$, force transfer rate between the two structures is not obvious, but in the high frequency band of $100 \sim 2500 \mathrm{~Hz}$, it is obvious that the force transfer ratio of bearing structure which has used the connection of resinous cast gasket is lower than the 
bearing housing structure with steel gasket. As it is shown in Tab 1, the bearing housing structure of the resinous cast gasket has better function of blocking dynamic force transfer and isolation effect of the force.

\section{Damping Characteristic Test of Resinous Cast Gasket}

Using resinous cast gasket between steady bearing and transition support of the rack, making contrast experiment on steady bearing which has been connected with steel gasket and that which has used the connection of three different models resinous cast gasket that produced by different manufacturer.

\section{Frequency-response Function Test}

Using excitation method by hammer, testing the dynamic characteristic of the steady bearing with the connection of steel gasket and three different models resinous cast gasket respectively in the condition of the rack. The arrangement of measuring points is as shown in Fig.5 and Fig.6.

Frequency response function curves of steady bearing with the connection of steel gasket and three different models resinous cast gasket has been obtained at the measuring points of \# 10 respectively. Analyzing the frequency response function curves, and plotting the effect of vibration isolation of three different models of the resinous cast gasket that compared with the steel gasket at the resonance frequency at the measuring points of \# 9 \#12, as shown in Fig.7.

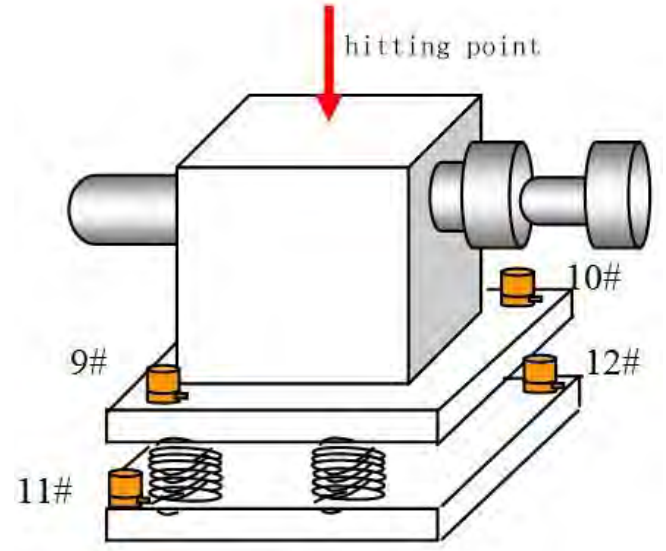

Fig. 5 Sketch Map of Testing Point Disposal

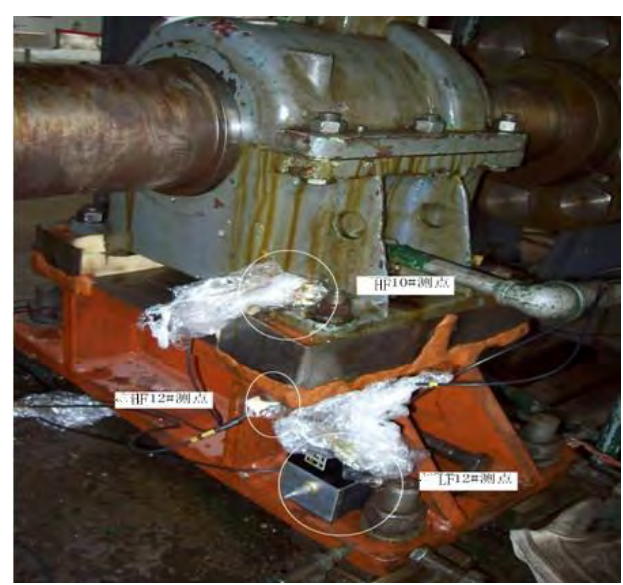

Fig. 6 Sketch Map of Testing Point

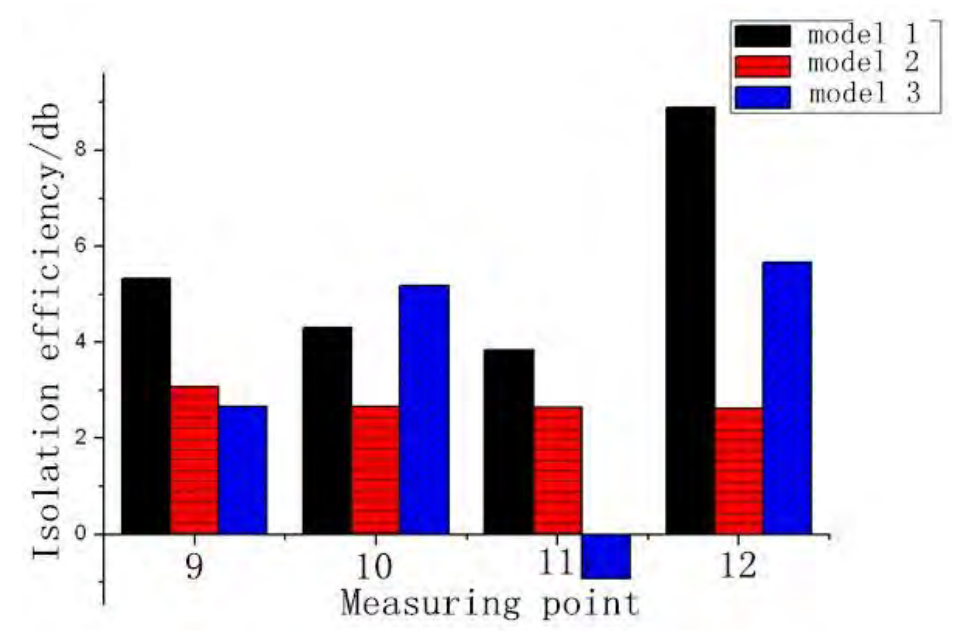

Fig. 7 Damping Efficiency of Different Resinous Cast Gasket

The analysis result of frequency-response function test shows that the damping efficiency which have been connected with three different models of resinous cast gaskets are obvious lower than that connected with steel gasket at the resonance frequency. Synthesize the test results of each measuring point. In general, the damping effect of resinous cast gasket of type 1 is the best. 


\section{Damping Performance Test of Rack}

The measuring points arrangement of damping performance test of rack is as shown in Fig.6. The shaft speed range is: $25 \mathrm{r} / \mathrm{min} \sim 400 \mathrm{r} / \mathrm{min}$.

The comparison of total amplitude and vibration attenuation quantity at the measuring points of \#10 is as shown in Fig.8 Fig.9.
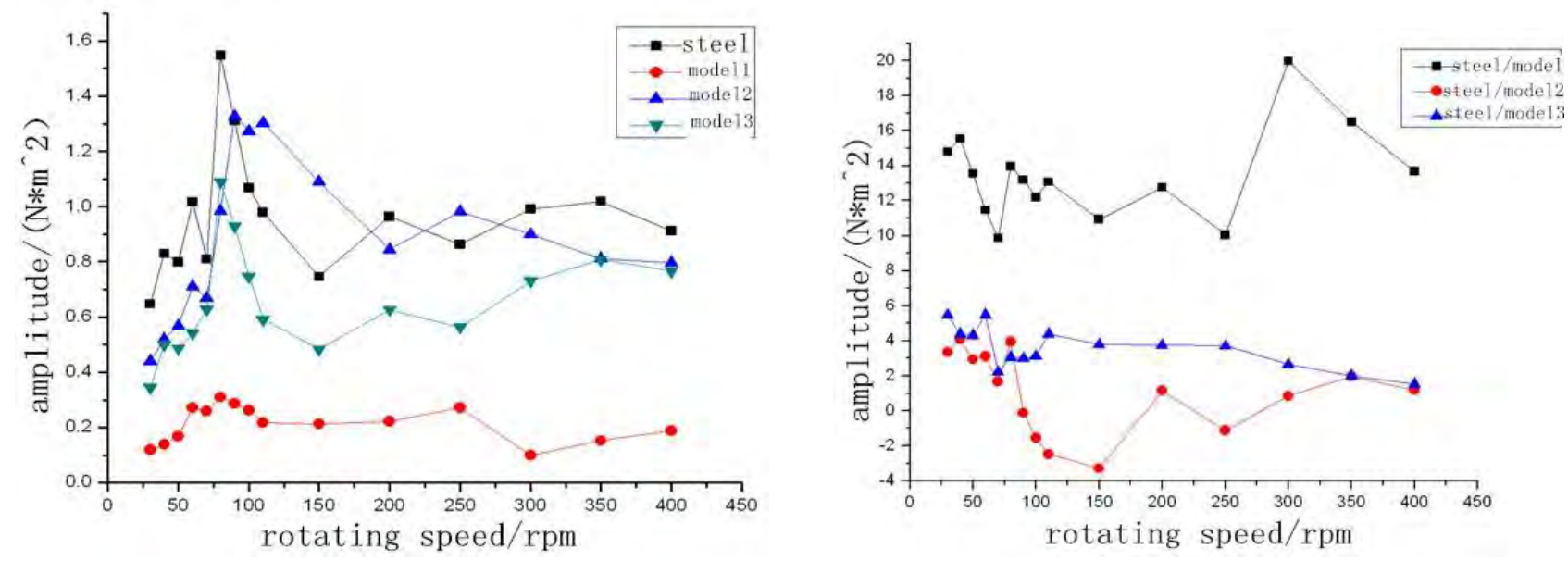

Fig. 8 Total Vibratory Extent of 10\# Testing Point Fig. 9 Damping Quantity of 10\# Testing Point

Under the working condition of the rotational speed, the total acceleration amplitude of steady bearings which have been connected with different models of resinous cast gaskets at high frequency measuring point is less than that connected with steel gasket on the whole, it has an obvious effect on the dumping of total acceleration amplitude at each measuring point, which the resinous cast gaskets of type 1 has the largest amount of the dumping of total acceleration amplitude. Although there are some fluctuations in some working conditions, it is better than the resinous gaskets of type 2 and type 3 .

\section{Summary}

According to the result of material characteristic testing, damping effect calculating and damping performance test of rack, it shows that using connection structure of resinous cast gaskets between shafting equipment and the hull has good function of blocking dynamic force transfer and damping effect, with type 1 is the best.

In this paper, according to the material damping characteristics analysis of connection structure of resinous cast gaskets, damping effect calculating and damping performance test of rack, figuring out a large number of experimental data. It provides a great guidance to the project practice, which laying a good technical foundation for further research for the optimization design and noise reduction performance of the connection between shafting and the hull.

\section{References}

[1]Guo Quanli, Yu Qiang, Li Tianyun. Study on damping properties of resin based damping material gasket[J]. Ship and ocean engineering, 2008, 37(4):46 49.

[2]LI T Y, LIU P F, ZHU X, WANG X. Pouring moulding characteristics and the relative mechanical properties of marine epoxy resin gasket[J]. Chinese Journal of Ship Research, 2012, (1):100 104.

[3]ZHANG W Y. Progress in development and application of damping materials[J]. Development and Application of Materials, 2011, (02):75 78. 